\title{
Over-Mentalisation and Delusions in Patients With Psychosis: A Meta-Analytic Review
}

\author{
Chen Zhu · Suzanne Ho-wai So \\ Department of psychology, The Chinese University of Hong Kong
}

\section{Introduction}

- Delusional beliefs typically involve intentional others (e.g., god, specific persons or organisations, etc.; Bell et al., 2017). Kapur (2003) hypothesised that psychosis is a state of aberrant salience, where delusions represent a top-down cognitive effort to make sense of the aberrantly salient experiences.

- In contrast to negative symptoms that are associated with a theory of mind deficit, delusions, particularly the paranoid subtype, appear to be associated with excessive attribution of others' mental states, even when the situation does not necessarily require so, namely, a tendency of over-mentalisation (OM; e.g., Bliksted et al., 2017; Montag et al., 2011).

- The current meta-analytic review aimed to collate current research on the relationship between over-mentalisation and delusions in patients with psychosis. A positive effect size between delusion severity and over-mentalisation was hypothesised.

\section{Methods}

\section{- Type of empirical studies}

measured over-mentalisation and reported its relationship with severity of delusions

data available upon request

\section{- Type of participants}

At least a group of adult patients (aged 18 or above) with schizophrenia spectrum and other psychotic disorders, and with either current or previous experience of delusions.

\section{- Other inclusion criteria}

published in a peer-reviewed journal

with English text available

\section{- Exclusion criteria}

\section{case studies or qualitative studies}

studies without enough information to compute the effect sizes either from their reports and upon request

- This review followed the PRISMA guidelines (Liberati et al., 2009).

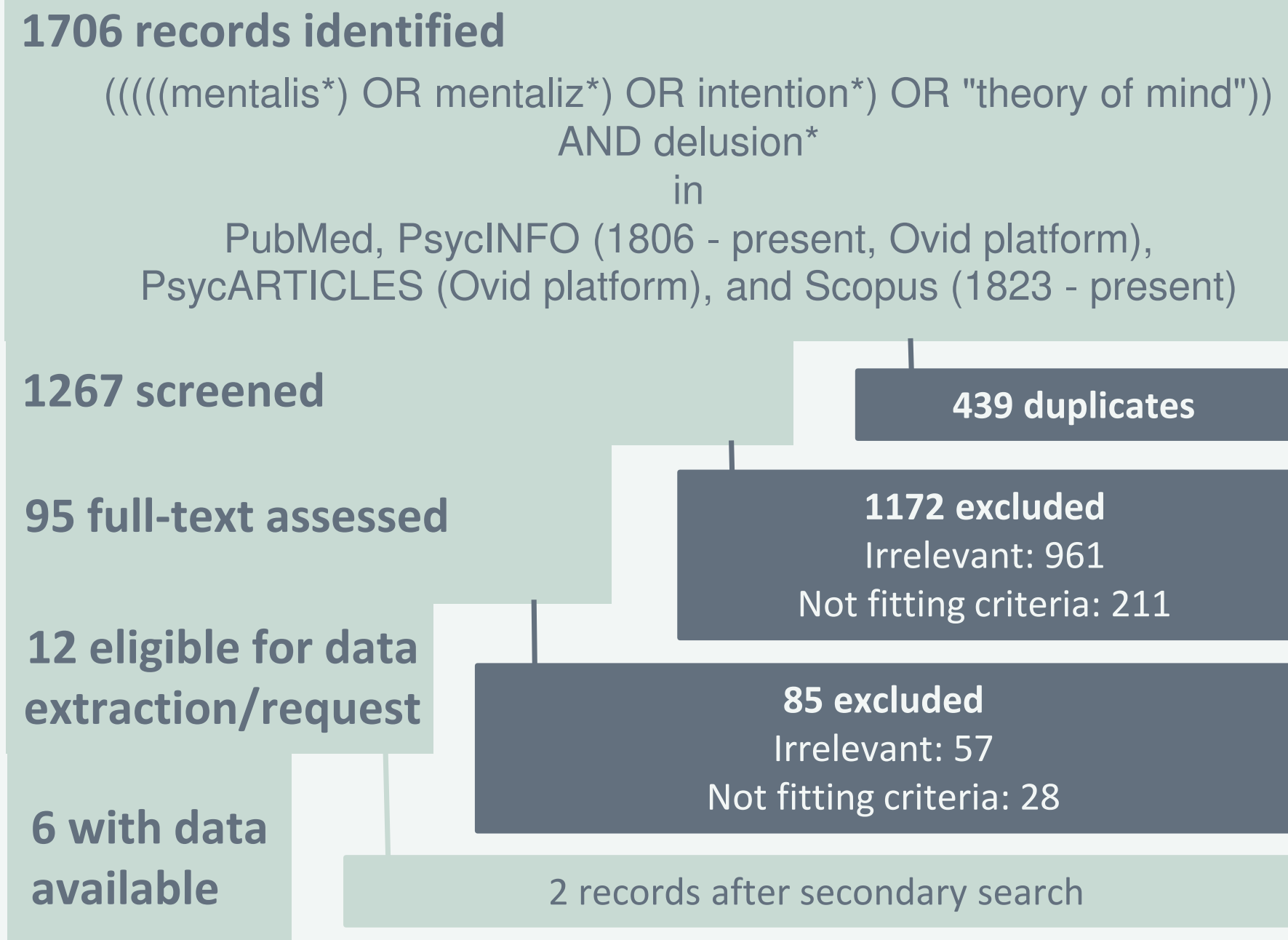

\section{Preliminary Results}

- Data were based on six studies (385 participants in total, see below table on the left). Primary inclusion criteria of included studies was a diagnosis of schizophrenia-spectrum disorder. Primary exclusion criteria were neurological disorder, head injury, substance misuse, and intellectual disability. A range of measures for over-mentalisation were included.

- Risk of bias within and across study was acceptable. As shown in the below table (on the right), Pearson rs suggested a positive association between over-mentalisation and severity of delusions, with effect sizes ranging from -0.18 to 0.37 .

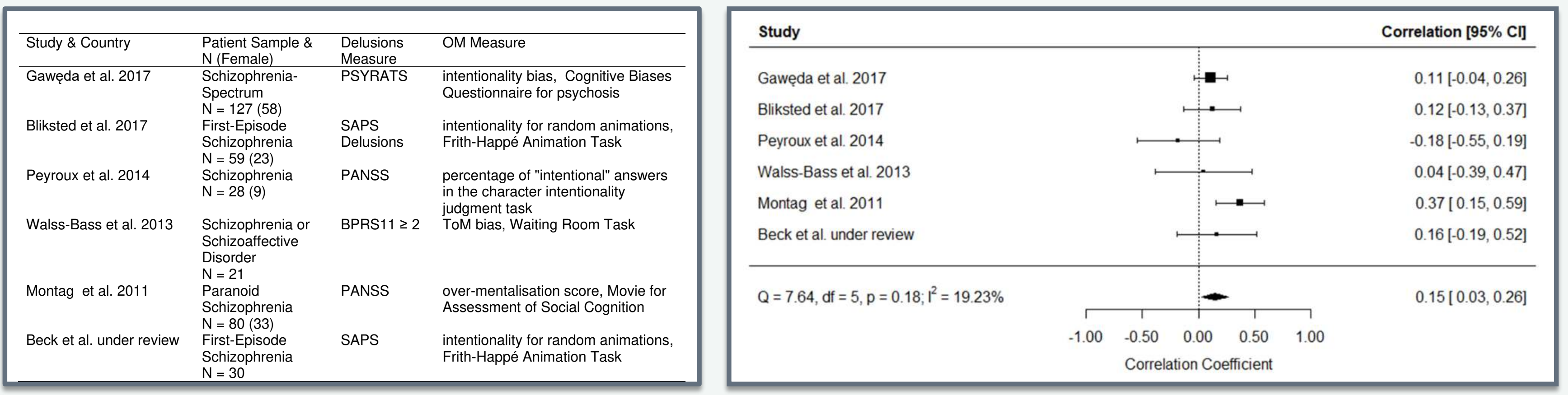

\section{Discussion}

- A small but significant effect size $(r=0.15)$ between over-mentalisation and severity of delusions was found, supporting the current hypothesis. However, results warrant future replication research considering the unavailability of some data at the time of analysis.

- Measures of over-mentalisation were heterogenous. We did not have enough data to compare effect sizes across studies that used different over-mentalisation measures.

- In light of co-occurrence and heterogeneity of psychotic symptoms, how over-mentalisation may be related to other positive and negative symptoms remained to be answered. Single-symptom approach could be considered. 